\title{
Idid: 一种基于免疫的动态入侵检测模型
}

\author{
李 涛 \\ (四川大学计算机学院, 成都 610065. E-mail: litao@scu.edu.cn)
}

\begin{abstract}
摘要 提出了一种新的基于免疫的动态入侵检测模型(Idid), 建立了入侵检测中有关自体、抗原的动态 描述方法, 提出了免疫细胞的动态耐受概念, 并建立了成熟细胞的生命周期以及免疫记忆等的动态模 型及其递推方程. 解决了计算机免疫系统中自体、非自体的动态描述问题, 并同时有效地克服了传统计 算机免疫系统中成熟细胞生成效率较低等缺陷. 对模型进行了仿真, 对比实验表明这种新型的入侵检 测模型较传统方法具有更好的适应性.
\end{abstract}

\section{关键词 入侵检测 人工免疫 耐受 免疫记忆 否定选择}

网络安全中的入侵检测问题 ${ }^{[1]}$ 与生物免疫系统 (biological immune system: BIS)消灭外来病原体入侵 问题具有惊人的相似性 ${ }^{[2]}$. BIS具有良好的多样性、耐 受性、免疫记忆、分布式并行处理、自组织、自学习、 自适应和鲁棒性等特点, 引起研究人员的普遍关注 [3 8].

1994 年Forrest等人 ${ }^{[9]}$ 提出了否定选择算法 (negative selection algorithm), 从而极大地刺激了计 算机免疫系统(computer immune system, CIS)的研究. Hofmeyr和Forrest等人 ${ }^{[10 ~ 12]}$ 为CIS提出了一种称之为 ARTIS的通用架构, 在该架构中, BIS的有关概念和机 制得到很好的体现，例如自体(self)、非自体(nonself)、 自体耐受(self-tolerance)、免疫细胞(检测器)、记 忆细胞(记忆检测器)、协同刺激等等. 目 前, 几乎 所有已发布的CIS其设计思路均源于ARTIS ${ }^{[2]}$, 例如 Dasgupta和Harmer等人 ${ }^{[13,14]}$ 在ARTIS的基础上, 利用 移动代理仿真免疫细胞对网络活动进行监控, 从而 提出了CIS的一种基于移动代理的实现框架.

然而, 现有的CIS存在两个缺陷: 其一是自体库 非常庞大, 例如, 在基于ARTIS的CIS应用LISYS ${ }^{[12]}$ 的实验过程中, Hofmeyr等人仅在 50 天的时间内就收 集了 230 多万个自体数据. 由于成熟检测器的训练代 价与自体集合的大小成指数关系 ${ }^{[15]}$, 从而使得直接 使用从网上收集自体进行未成熟检测器的自体耐受 工作变得不可行. 迫不得已, LISYS的实验目标最终 降低为 7 种网络攻击的检测问题, 并对网络提供的服 务及正常的网络活动等进行简化以减少自体的数目, 同时经过艰苦、繁杂的分类工作后, Hofmeyr最后选定 某几个时间段内的 3900 多个自体用于检测器的耐受
处理, 在一定程度上缓解了检测器耐受工作的训练 代价. 然而, 即便如此, 其计算代价依然不小.

另一缺陷是系统中自体/非自体的描述为一种静 态方式, 一旦定义后就极少变化. 然而在具体应用中, 自体/非自体一方面难以准确定义, 另一方面它们之 间经常发生角色变化, 因此, 随着时间的推移需要及 时地修正, 例如今天看来是正常的网络行为, 可能在 明天就会被确认为非法的网络攻击; 反之亦然. 静态 自体/非自体的描述模型, 不能满足真实网络环境下 网络监控的需求, 缺乏较好的适应能力.

上述两个问题已成为CIS具体应用中的主要障碍. 动态克隆选择算法 ${ }^{[16]}$ 以及文献[17]试图改变这一现 状, 并进行了初步尝试, 但由于其自体的更新周期过 快(仅为一个检测周期), 几乎很难从网络上收集到较 为恰当的自体元素, 并且缺乏系统性的描述, 方法也 较为粗粘, 离具体的应用尚有较长的距离. 另外, 现 有的CIS模型几乎均缺乏严格的定量描述，导致其实 现具有较大的随意性, 不利CIS的推广应用.

本文基于免疫原理, 提出了一种新的动态入侵 检测模型(an immune based dynamic intrusion detection: Idid), 建立了入侵检测中有关自体、抗原的动态 演化数学模型, 提出了自体的自然动态定义、免疫细 胞的动态耐受概念, 并建立了成熟细胞的生命周期 以及免疫记忆等动态模型及其递推方程. 解决了传 统 CIS 中自体、非自体的动态描述和演化, 以及成熟 细胞生成效率较低等问题, 对模型进行了仿真以及 对比实验, 结果显示Idid较传统方法具有更好的适应 能力. 


\section{1 模型理论}

定义抗原 ${ }^{1}$ 集合 $A g \subset D, \quad D=\{0,1\}^{l}(l>0)$, 其中 $A g$ 为 IP 地址、端口号、协议类型、 TCP/UDP/ICMP 域、IP 包的长度等标志网络事务特征的二进制串. 定 义自体集合 Self 为正常的网络服务, 非自体集合 Nonself 为来自网络的攻击, 有 Self $\cup$ Nonself $=A g$, Self $\cap$ Nonself $=\Phi$. 定义待检抗原集合 $s A g \subset A g$, $|s A g|=\eta *|A g|, \quad 0<\eta<1$. 定义免疫细胞 ${ }^{2}$ 集合 $B=$ $\{\langle d$, age, count $\rangle \mid d \in D$, age, count $\in N\}$, 其中 $d$ 为抗体, age 为细胞的年龄, count 为匹配数, $N$ 为自然数集合, $d$, age 和 count 又称为免疫细胞的域. 为引用方便, 定 义免疫细胞 $x$ 的下标运算符“ . ” x.fieldname $=x$ 的域 fieldname 的值.

给定 $B=M_{b} \cup T_{b}, \quad M_{b} \cap T_{b}=\Phi$, 其中 $M_{b}=$ $\{x \mid x \in B, x$.count $\geq \beta, \forall y \in \operatorname{Self}\langle x . d, y\rangle \notin$ Match $\}$ 为 记忆免疫细胞集合, $\beta(>0)$ 为激活國值, $T_{b}=$ $\{x \mid x \in B, x$. count $<\beta, \forall y \in$ Self $\langle x . d, y\rangle \notin$ Match $\}$ 为 成熟免疫细胞集合, 即: 成熟免疫细胞为不与自体匹 配且其抗原匹配数还未达到激活阈值的细胞(还未被 激活), 而记忆免疫细胞为成熟细胞中抗原匹配数超 过激活阈值的细胞 (已被激活过), 其中 Match = $\left\{\langle x, y\rangle \mid x, y \in D, f_{\text {match }}(x, y)=1\right\}$ 为 $D$ 中的匹配关系， $f_{\text {match }}(x, y)$ 的取值取决于 $x$ 与 $y$ 之间的匹配度 (亲和力): 若其大于一给定的阈值, 则为 1 否则为 0 . 本模型中, 亲和力可以为Euclidean距离, Manhattan距离, Hamming距离, r连续位(r-contiguous bites)匹配, 等等 ${ }^{[2]}$. 定义 $I_{b}=\{\langle d, a g e\rangle \mid d \in D, a g e \in N\}$ 为末成熟免疫细胞 集合.

图 1 为 Idid 的体系架构, 其中新生的未成熟细胞 需经历一个自体耐受期, 若在耐受期间匹配自体, 则 会走向死亡(否定选择). 未成熟细胞经历耐受期后成 熟进化为成熟细胞, 并由此步入成熟细胞生命周期: 成熟细胞仅有固定长度的生命周期, 若在其生命周 期内没有匹配到足够的抗原, 则会走向死亡(老死); 否则进入激活状态. 在激活状态下, 若缺乏协同刺激, 则表明被检测到的抗原为自体抗原, 也就是发生错 误肯定(false positive error), 意味着该细胞是一个错
误的免疫细胞, 需要立即清除; 若获得协同刺激, 则 表明被检测到的抗原确系非自体抗原, 于是将该细 胞进化为记忆细胞. 记忆细胞具有无限长的生命周 期, 一旦匹配到一个抗原, 则会被立即激活.

网络中传输的 IP 包经抗原提呈后, 形成一特定 的抗原集合 $A g$ (表达网络特性的二进制串). 模型的 任务为对输入的抗原集合 $A g$, 分 $\delta$ 步 ( $\delta>0$, 常数), 每步从 $\mathrm{Ag}$ 中选出一定数量的抗原组成待检抗原集合 $s A g$, 通过检测细胞 $B$ (记忆细胞或成熟细胞) 将之分 类为自体 $A g_{\text {Self }}$ 或非自体 $A g_{\text {Nonselfs }}$ ，其中被分类为自体 的抗原 $A g_{\text {Sell }}$ 被并入整个模型的自体集 Self 中, 用作 下一步未成熟细胞的耐受处理. 由于 Self 是动态的, 因此耐受也是动态的: 即系统中可能同时出现对某 一种自体耐受和不耐受的细胞. Idid 通过协同刺激解 决此类问题.

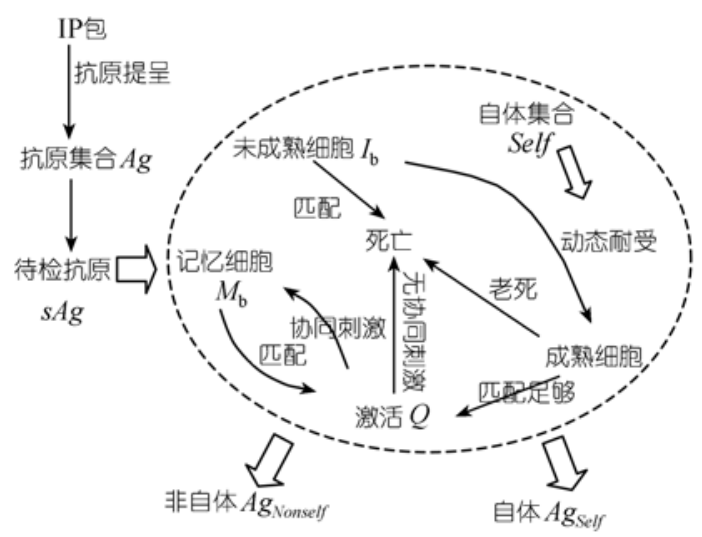

图 1 Idid 体系架构

下面我们采取集合代数的描述方法, 分别定量 描述模型中有关自体集合 $S e l f$, 抗原集合 $A g$ ，未成熟 细胞集合 $I_{b}$, 成熟细胞集合 $T_{b}$, 记忆细胞集合 $M_{b}$ 随 时间动态演化的过程.

\section{1 动态自体模型}

$$
\operatorname{Self}(t)=\left\{\begin{array}{lrl}
\left\{x_{1}, x_{2}, \ldots, x_{n}\right\}, & t & =0 \\
\operatorname{Self}(t-1), & & \bmod \delta \neq 0 \\
\operatorname{Self}(t-1)-\operatorname{Self}_{\text {dead }}(t) & \cup A g(t-1), \\
& & >0 \wedge t \text { mod } \delta=0
\end{array}\right.
$$

1) 本文关于抗原的分类采用理论免疫学的分类方法, 即抗原分为自体抗原和非自体抗原, 为方便起见, 又简称为自体和非自体

2) 这里的免疫细胞系仿真 BIS 中的免疫细胞如 B 细胞或 $T$ 细胞等, , 在某些 CIS 的文献中也被称为检测器、淋巴细胞等 


$$
\operatorname{Self}_{\text {dead }}(t)=\left\{\begin{array}{l}
\phi, \quad|\operatorname{Self}(t-1)|+|\operatorname{Ag}(t-1)|<L \\
\{\operatorname{Self}(t-1) \text { 中最早 } \\
\text { 收集的 }|\operatorname{Ag}(t-1)| \quad \text { otherwise } \\
\text { 个元素 }\},
\end{array}\right.
$$

方程 (1) 模拟了自体的动态演化情况, 其中 $x_{i} \in D(i \geq 1, i \in N)$ 为初始自体集合, $\delta(>0)$ 为自体的 演化周期(参见 1.2 抗原演化模型, 注意在 1.2 节中, 针对抗原, $\delta$ 又称为抗原更新周期), 所谓自体的演化 周期意思是自体集合以 $\delta$ 为周期进行演变：在 $\delta$ 周期 内, 自体集合 Self 维持不变, 在 $\delta$ 周期结束后, 将补 充进新的自体元素, 即由上一阶段从网上收集的抗 原集合 $A g(t-1)$ 中通过免疫细胞(记忆细胞和成熟细 胞)经过 $\delta$ 个周期检测后剩余的抗原，即补充被分类 为自体的集合 $A g_{\text {Self }}$. 同时，当自体集合大小超过阈 值 $L$ 时, 按先进先出的原则淘汰一部分自体元素 Self $f_{\text {dead }}$, 不致使整个自体集合的大小无限制地增长, 从而确保免疫耐受工作能够以高效率的方式进行(参 见 1.3 动态耐受模型).

动态自体模型能够很自然地描述真实网络环境 下自体随时间动态演化的情形，例如系统管理员决 定临时开放一些新的端口, 提供一些新的服务, 或者 取消某些网络服务, 等等.

动态自体模型的核心是自体的自然动态定义方 法: 自体的来源为通过在线收集来自网络上某一段 时间内(自体演化周期)、并经过免疫细胞过滤后的正 常网络活动行为特征, 并形成相应的、供未成熟细胞 进行耐受处理的自体库(参见 1.3 动态耐受模型). 在 自体演化周期内, 自体集合相对稳定, 或者说, 自体 集合以自体演化周期为节拍与真实网络计算环境同 步演化, 从而做到了变与不变的有机统一, 确保了模 型的可操作性.

虽然每一步 Idid 仅收集了一小部分自体行为, 但随着时间的推移, $\bigcup_{t=0}^{\infty} \operatorname{Self}(t)$ 将覆盖整个自体空间, 于是, 我们有:

定理 1 动态自体模型将从时间上覆盖整个自 体空间，或者说，其自体描述从宏观上是完备的.

动态自体模型基于真实网络环境下的一个概率 结果 ${ }^{[1]}$ : 网络活动是连续的, 即刚刚发生的网络行为 有可能马上再次发生; 而突然出现的网络活动很可 能意味着网络攻击事件.

\section{2 抗原演化模型}

$$
\begin{gathered}
A g(t)= \begin{cases}\operatorname{Self}(0), & t=0 \\
A g(t-1)-A g_{\text {Nonself }}(t), & t>0, t \bmod \delta \neq 0 \\
A g_{\text {new }}(t), & t>0, t \bmod \delta=0\end{cases} \\
\operatorname{sAg}(t) \subset A g(t), \quad|s A g(t)|=\eta *|A g(t)|, \quad t \geq 0
\end{gathered}
$$

其中 $\delta$ 为抗原更新周期, 表示每 $\delta$ 代, 就把 $A g$ 换为全新的抗原集合 $\left(A g_{\text {new }}\right) . s A g$ 为系统每次进行处 理的抗原, 其元素按比例 $\eta(0<\eta \leq 1)$ 随机从 $A g$ (由自 体和非自体元素组成)中抽取, $\eta$ 为检测系数. $A g_{\text {Nonself }}$ 为 $t$ 时刻被检测出来的非自体抗原, $M_{\text {new }}(t)$ 为 $t$ 时刻新 生的记忆细胞(参见 1.5 动态免疫记忆模型).

更新周期内抗原集合的变化只是删除被检测出 来的非自体抗原, 这样通过 $\delta$ 步的检测, 剩下的抗原 即被分类为自体抗原 $A g_{\text {Self }}$, 并送给未成熟免疫细胞 $I_{b}$ 进行耐受处理(参见 1.1 动态自体模型, 1.3 动态耐 受模型).

初始时抗原集合为初始的自体集 $\operatorname{Self}(0)$, 此时 $B$

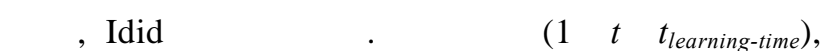
抗原集合为保持一定比例的自体、非自体, 以确保训 练出一定数量满足要求的成熟细胞和记忆细胞.

\section{3 动态耐受模型}

$$
\begin{gathered}
I_{b}(t)= \begin{cases}\left\{x_{1}, x_{2}, \ldots, x_{\xi}\right\}, & t=0 \\
I_{\text {tolerance }}(t)-I_{\text {maturation }}(t) \cup I_{\text {new }}(t), & t \geq 1\end{cases} \\
I_{\text {tolerance }}(t)=\left\{y \mid y \in I_{b}, y . d=x . d, y . \text { age }=x . \text { age }+1,\right. \\
x \in\left(I_{b}(t-1)-\left\{x \mid x \in I_{b}(t-1),\right.\right. \\
\exists y \in \operatorname{Self}(t-1)\langle x . d, y\rangle \in \text { Match }\})\} \\
I_{\text {maturation }}(t)=\left\{x \mid x \in I_{\text {tolerance }}(t), x . \text { age }>\alpha\right\} \\
I_{\text {new }}(t)=\left\{y_{1}, y_{2}, \ldots, y_{\xi}\right\}
\end{gathered}
$$

方程(6)模拟免疫细胞在骨髓中的生长情况, 其 中 $x_{i}=\langle d, 0\rangle(d \in D, 1 \leq i \leq \xi)$ 为初始随机生成的未成 熟免疫细胞, $\xi>0$, 为常数. $I_{\text {tolerance }}$ 为对上一阶段的自 体 $\operatorname{Self}(t-1)$ 经历一次耐受后剩下的免疫细胞, $\alpha \geq 1$ (常数)模拟耐受期, 未成熟细胞必须在动态耐 受模型中通过否定选择(如方程(7)所示, 删除那些识 别自体的未成熟细胞), 并经历一个周期为 $\alpha$ 的耐受 期方可成熟. $I_{\text {maturation }}$ 为 $t$ 时刻已经成熟的免疫细胞. $I_{\text {new }}$ 为 $t$ 时刻随机产生的新的未成熟免疫细胞, 其中 $y_{i}=\langle d, 0\rangle(d \in D, 1 \leq i \leq \xi)$. 
由于系统中自体的个数最多不超过 $L($ 参见 1.1 动 态自体模型), 因此, 动态耐受模型可以高效率地产 生成熟细胞.

不失一般性, 考虑极端情况, 系统中自体的数目 $|\operatorname{Self}(t)|=L(0<t \leq \infty)$, D’haeseleer等人 ${ }^{[15]}$ 指出, 对任意 的匹配规则, 生成固定数目的成熟细胞的时间复杂 性为: $O\left(\frac{-\ln \left(P_{f}\right)}{P_{m} \cdot\left(1-P_{m}\right)^{L}} \cdot L\right)$, 空间复杂性为: $O(l \cdot L)$, 其中, $P_{m}, P_{f}$ 分别表示匹配率和错误否定率, $l$ 为常数. 对不受候选细胞集合约束的细胞来说, $P_{f} \approx$

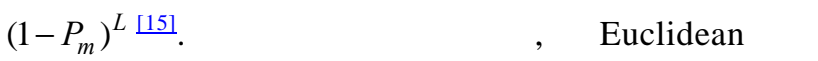
等 ${ }^{[2]}$, 其匹配率 $P_{m}$ 为常数, 于是, 生成固定数目的成 熟细胞的时间复杂性为: $O\left(\frac{-\ln \left(P_{f}\right)}{P_{m} \cdot\left(1-P_{m}\right)^{L}} \cdot L\right)=$ $O\left(\frac{-\ln \left(1-P_{m}\right)^{L}}{P_{m} \cdot\left(1-P_{m}\right)^{L}} \cdot L\right)=O\left(\frac{-\ln \left(1-P_{m}\right)}{P_{m} \cdot\left(1-P_{m}\right)^{L}} \cdot L^{2}\right)=O(c), \quad$ 其 中 $c=\frac{-\ln \left(1-P_{m}\right)}{P_{m} \cdot\left(1-P_{m}\right)^{L}} \cdot L^{2}$ 为常数; 其空间复杂性为: $O(l \cdot L)=O\left(c^{\prime}\right)$, 其中 $c^{\prime}=l \cdot L$ 为常数. 上述结论是在 最坏情况下获得的. 于是, 综合考虑我们有:

定理 2 动态耐受模型产生成熟细胞的时空代 价小于一给定的常数.

另外, 由于 $I_{n e w}$ 的随机性, 从而使得新生成的成 熟免疫细胞具有较好的多样性. 并且, 在耐受过程中, 由于自体集合 Self 随时间动态变化, 因此, 相应的耐 受也是动态的, 即系统中可能同时出现对某一种自 体耐受和不耐受的细胞, 此类矛盾由协同刺激解决 (参见 1.5 动态免疫记忆模型), 错误的细胞在缺乏协 同刺激的情况下最终将会走向死亡.

虽然每一步系统产生的成熟细胞只能对某一部 分自体耐受, 但是在协同刺激的帮助下, 未经充分耐 受或发生变异的细胞(过去被禁止的网络活动, 现在 被允许, 如开放新的端口或服务等)将最终被清除(参 见 1.5 动态免疫记忆模型). 这样, 依据抗体指令系统 的完备性理论 ${ }^{[18]}$, 动态耐受模型将最终产生有效覆 盖整个抗原空间的抗体，或者说:

定理 3 动态耐受模型将最终产生识别所有抗 原的成熟细胞.

动态耐受模型很好地模拟了真实网络应用环境 中的网络活动情况: 一般来说, 经常出现的活动是正
常、合法的网络行为; 而突然出现的某种网络活动非 常可疑, 很可能是非法的入侵 ${ }^{[1]}$. 动态耐受模型被设 计成刚好保证新生的成熟细胞对那些经常性的正常 网络活动耐受, 从而确保了对突发事件检测的敏感 性. 由于突发的网络事件往往意味着网络攻击事件 的发生, 这就是说, 动态耐受模型生成的成熟细胞具 有很好的有效性.

1.4 成熟细胞生命周期

$$
\begin{aligned}
& T_{b}(t)= \begin{cases}\Phi, & t=0 \\
T_{b}^{\prime}(t) \cup T_{\text {new }}(t)-T_{\text {activation }}^{\prime}(t)-T_{\text {dead }}(t), & t \geq 1\end{cases} \\
& T_{b}^{\prime}(t)=T_{b}^{\prime \prime}(t)-P(t) \cup P^{\prime}(t) \\
& T_{b}^{\prime \prime}(t)=\{y \mid y \in B, y . d=x . d, y . \text { age }=x . \text { age }+1, \\
& \left.y \text {.count }=x \text {.count, } x \in T_{b}(t-1)\right\} \\
& P(t)=\left\{x \mid x \in T_{b}^{\prime \prime}(t), \exists y \in \operatorname{sAg}(t-1) \quad\langle x . d, y\rangle \in \text { Match }\right\} \\
& P^{\prime}(t)=\{y \mid y \in B, y . d=x . d, y . \text { age }=x . \text { age, } \\
& y \text {.count }=x \text {.count }+1, x \in P(t)\} \\
& T_{\text {new }}(t)=\{y \mid y . d=x . d, y . \text { age }=0, y . \text { count }=0, \\
& \left.y \in B, x \in I_{\text {maturation }}(t)\right\} \\
& T_{\text {activation }}^{\prime}(t)=\left\{x \mid x \in T_{b}^{\prime}(t), \text { x.count } \geq \beta\right\} \\
& T_{\text {activation }}(t)=\{y \mid y \in B, y . d=x . d, y . \text { age }=0, \\
& \left.y \text {.count }=x \text {.count, } x \in T_{\text {activation }}^{\prime}(t)\right\} \\
& T_{\text {dead }}(t)=\left\{x \mid x \in T_{b}^{\prime}(t), x \text {.age }>\lambda \wedge x \text {.count }<\beta\right\}
\end{aligned}
$$

方程(10)模拟成熟免疫细胞的生命周期. 免疫细 胞成熟后, 必须在生命周期 $\lambda(>0)$ 内与抗原结合, 且 累计足够的亲和力(匹配足够的抗原, $\beta>0$ ), 从而进 入激活状态 $\left(T_{\text {activation, }}\right.$, 其中 $T_{\text {activation }}^{\prime}$ 是即将被激活的 细胞), 否则, 将走向死亡 $\left(T_{\text {dead }}\right)$, 被新生的成熟免疫 细胞所代替 $\left(T_{n e w}\right) . P(t) 、 P^{\prime}(t)$ 模拟成熟细胞匹配非自体 抗原的过程, $T_{b}^{\prime \prime}(t)$ 模拟细胞年龄增长, $T_{b}^{\prime}(t)$ 模拟细 胞的一代演化过程.

\section{5 动态免疫记忆模型}

$$
\begin{gathered}
M_{b}(t)= \begin{cases}\Phi, & t=0 \\
M_{b}(t-1)-M_{b}^{\prime}(t) \cup M_{n e w}(t), & t \geq 1\end{cases} \\
M_{b}^{\prime}(t)=\left\{x \mid x \in M_{b}(t-1), \exists y \in s A g(t-1)\right. \\
\langle x . d, y\rangle \in \text { Match }\} \\
M_{\text {activation }}(t)=\{y \mid y \in B, y . d=x . d, y . \text { age }=0, \\
\text { y.count } \left.=x . \text { count }+1, x \in M_{b}^{\prime}(t)\right\}
\end{gathered}
$$




$$
\begin{gathered}
Q(t)=\left\{\begin{array}{l}
\Phi, \\
Q^{\prime}(t) \cup M_{\text {activation }}(t) \cup T_{\text {activation }}(t) \\
-M_{\text {new }}(t)-Q_{\text {dead }}(t),
\end{array} \quad t>0\right. \\
Q^{\prime}(t)=\{y \mid y \in B, y . d=x . d, y . \text { age }=x . \text { age }+1, \\
y . \text { count }=x \text {.count, } x \in Q(t-1)\} \\
Q_{\text {dead }}(t)=\left\{x \mid x \in Q^{\prime}(t), x . \text { age } \geq \rho\right\} \\
M_{\text {new }}(t)=\left\{x \mid x \in Q^{\prime}(t)-Q_{\text {dead }}(t), f_{\text {costimulation }}(x)=1\right\} \\
f_{\text {costimulation }}(x)= \begin{cases}1, & \text { 针对 } x \text { 有外部信号 } \\
0, & \text { otherwise }\end{cases}
\end{gathered}
$$

方程(19)刻画了记忆细胞的动态变迁情况. 记忆 细胞具有无限长的生命周期, 一旦匹配一个抗原 $\left(M_{b}^{\prime}\right)$, 将会立即进入激活状态 $M_{\text {activation }}$.

从记忆细胞或成熟细胞进入激活状态后的细胞 $Q$ 在协同刺激 $f_{\text {costimulation }}$ (外部系统的输入信号或网络 安全管理员的应答等等)的帮助下将分别重新转变或 进化为记忆细胞 $M_{\text {new }}$, 以完成一次免疫应答的过程. 若进入激活状态后的细胞在协同刺激周期 $(\rho>0)$ 内缺 乏协同刺激, 意味着该细胞错误地将自体抗原分类 为非自体, 即发生错误肯定, 则该细胞 $Q_{\text {dead }}$ 将被淘 汰.

$Q^{\prime}$ 模拟激活细胞的一代演化(细胞年龄增长), 其中 y. age 在这里意思是细胞 $y$ 有多久没有接收到协 同刺激.

协同刺激并非与细胞的激活同步进行, 必须给 外部系统足够的时间, 这一点对于外部信号为网络 安全管理员的应答尤为重要.

协同刺激的方式也可以采取与前面(方程(25))刚 好相反的形式: 若被激活的细胞在协同刺激周期内 接收到协同刺激, 意味着该细胞错误地将自体抗原 分类为非自体, 则强行淘汰该细胞(令其死亡); 否则, 表明被该细胞检测到的抗原确系非自体，则促使其 产生成功的免疫应答, 并使之重新进入(对记忆细胞 的激活体而言)或进化为(对成熟细胞的激活体而言) 记忆细胞, 开始新的循环.

如同 BIS 一样, Idid 也有两种类型针对抗原的免 疫应答: 初次应答和二次应答, 分别由成熟细胞和记 忆细胞发出. 成熟细胞的初次应答需要一个较长的 学习过程: 首先需要一段时间以产生合适的未成熟 细胞, 然后该未成熟细胞需在动态耐受模型中历经 $\alpha$
个耐受期后方可成熟(参见方程(7)和(8)), 成熟后还 必须累计足够的亲和力方能被激活(参见方程(16)和 (17)), 因此初次应答效率相对较低. 在此学习过程中, 那些对抗原分类没有作用或作用不大的细胞将被淘 汰(参见方程(18)), 优势细胞(对抗原具有良好分类作 用的细胞)将会被保留并进化为记忆细胞, 以便当类 似抗原二次入侵时能进行更高效的应答 (二次应答). 二次应答由记忆细胞发出, 迅速、强壮, 无需学习过 程, 一旦记忆细胞与抗原发生匹配, 将会被立即激 活、响应(参见方程(20)和(21)).

\section{2 模型仿真}

实验在四川大学网络安全攻防实验室进行, 总 计 40 台计算机参与了实验. 取 32 位源、目的 IP 地 址、16 位端口以及 16 位协议标志位等构成 $l=96$ 的 二进制串提呈抗原, 采用 $r$ 连续位匹配方法计算亲和 力 $(r=8)$. 选取初始自体集合大小 $n=40$, 每步产生 $\xi=$ 10 个未成熟细胞. 每次从网上捕获 100 个 IP 包, 对 IP 包进行预处理, 将其变换为检测系统处理的抗原 格式, 取检测率 $\eta$ 为 0.8 . 模拟对网络进行 syn flood, land, smurf, teardrop 等 20 多种攻击, 其中非自体包和 自体包的比例为 9:1. 实验结果采用 $\mathrm{TP}$ 值(检测率) 和 FP 值(误报率)对模型进行评估.

模型参数的选取一般依据真实网络环境中的具 体情况. 由于模型的自体定义为一种动态方式, 耐受 期 $\alpha$ 一般考虑为 $1 \sim 2$ 天, 生命周期 $\lambda$ 应比 $\alpha$ 大, 抗原 更新周期 $\delta$ 的选取一般以线速条件下不丢包为宜, 自体集合大小 $L$ 的选取主要依据当前机器的物理条 件, 在不影响整个系统的效率的情况下, 可以考虑选 择较大的 $L$, 细胞的激活阈值 $\beta$ 的选择可参考文 献[10]. 各种参数下的实验结果如图 2 6 所示, 实验 显示: (i ) 如图 2 所示, 当激活阈值 $\beta$ 较小时, 由于 成熟细胞未经充分学习而被激活, 因此相对增大了 误报率; (ii) 如图 3 所示, 当耐受期 $\alpha$ 较小时, 由于 未成熟细胞未经充分耐受, 从而导致了较高的 FP 值; (iii) 图 4 显示, 过小的生命周期 $\lambda$ 将导致较低的检测 率 TP, 这是由于成熟细胞因为没有足够的时间(生命) 等待其期望的非自体元素(网络攻击)造成的, 然而, 较大的 $\lambda$ 也将导致较大的 FP 值, 因此需要综合考虑; 生命周期 $\lambda$ 的设置主要与人们的网络活动周期有关, 般地, 真实网络环境中正常网络活动的变化周期 
为 1 个星期(7 天); (iv) 图 5 显示 $\delta$ 对 TP, FP 值的影 响较小, 这与期望的一致; ( $\mathrm{V}$ ) 如图 6 所示, 自体集 合的大小 $L$ 对 FP 有较大的影响, 这是因为 $L$ 越小, 未 成熟细胞自体耐受越不充分, 从而致使成熟细胞识 别自体的概率增加(FP).

实验结果表明, 当 $\beta=40, \alpha=48 \mathrm{~h}, \lambda=7 \mathrm{~d}, \delta=$ 15 代, $L=2500$ (如图 7 所示), 获得较为满意的 TP 值

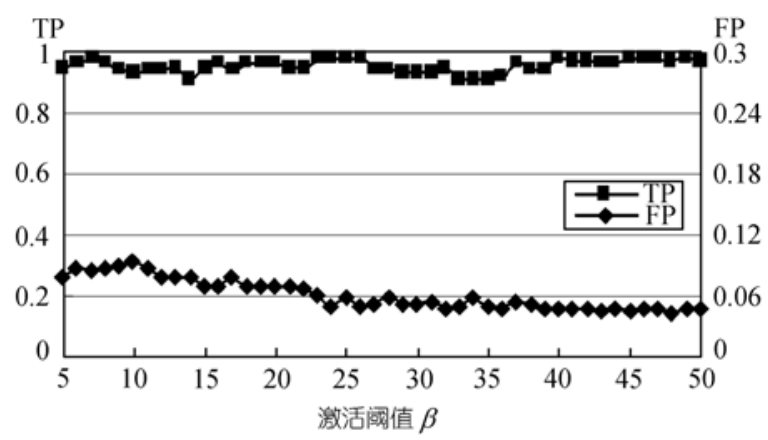

图 2 激活阈值 $\beta$ 对模型 TP 值和 FP 值的影响 其中 $\alpha=48 \mathrm{~h}, \lambda=7 \mathrm{~d}, \delta=15$ 代, $L=2500$ 个

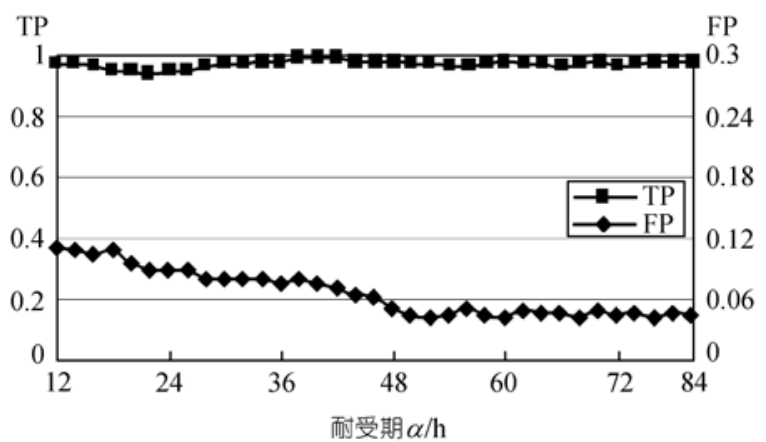

图 3 耐受期 $\alpha$ 对模型 TP 值和 FP 值的影响 其中 $\beta=40, \lambda=7 \mathrm{~d}, \delta=15$ 代, $L=2500$ 个

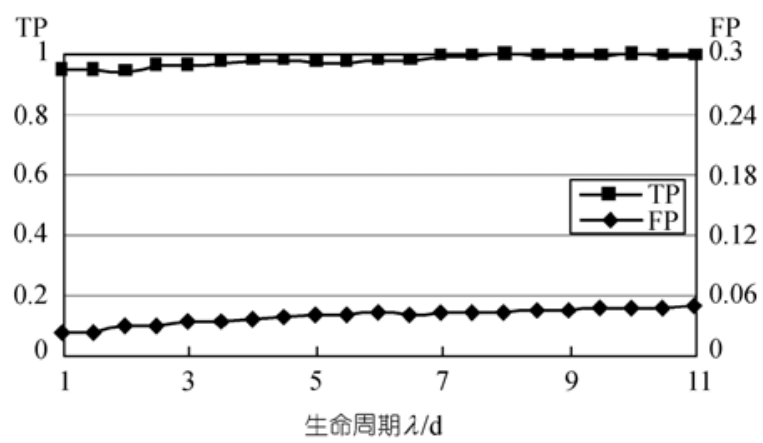

图 4 生命周期 $\lambda$ 对模型 TP 值和 FP 值的影响 其中 $\beta=40, \alpha=48 \mathrm{~h}, \delta=15$ 代, $L=2500$

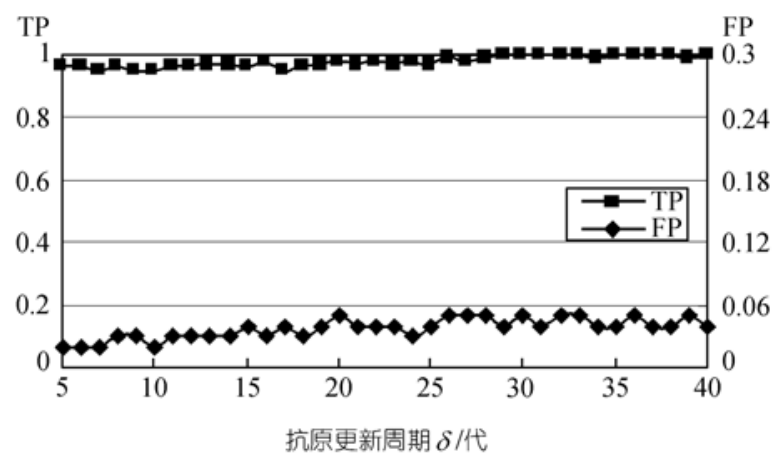

图 5 抗原更新周期 $\delta$ 对模型 TP 值和 FP 值的影响 其中 $\beta=40, \alpha=40 \mathrm{~h}, \lambda=7 \mathrm{~d}, L=2500$ 个

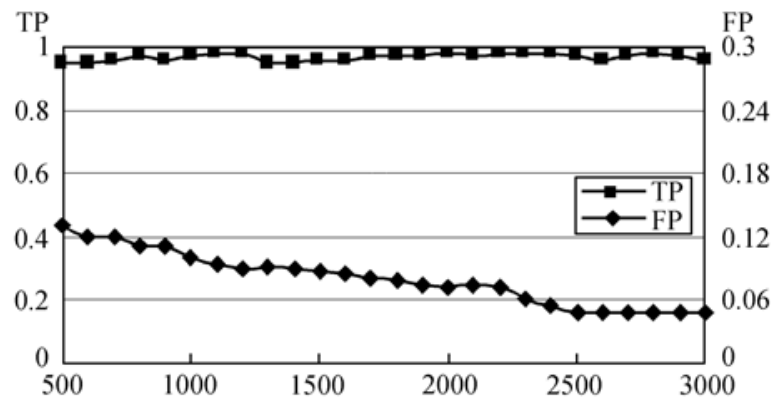

自体集合 $L /$ 个

图 6 自体集合 $L$ 对模型 TP 值和 FP 值的影响 其中 $\beta=40, \alpha=48 \mathrm{~h}, \lambda=7 \mathrm{~d}, \delta=15$ 代

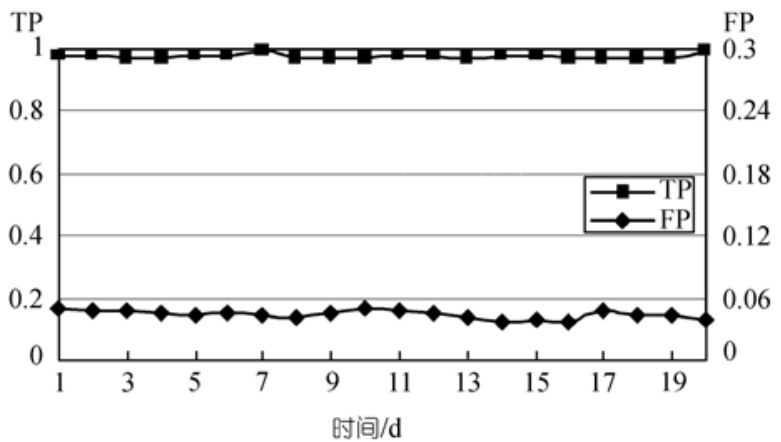

图 $7 \beta=40, \alpha=48 \mathrm{~h}, \lambda=7 \mathrm{~d}, \delta=15$ 代, $L=2500$ 个时系 统的工作情况

和 $\mathrm{FP}$ 值 $(\mathrm{FP}<5 \%, \mathrm{TP}>90 \%)$.

为检验Idid的性能, 我们还进行了有针对性的对 比实验. 对比对象为 Forrest 等人 ${ }^{[9,19]}$ 提出的 Exhaustive算法, Exhaustive算法是传统CIS的典型代 表, 对后来CIS的设计产生了重要的影响.

1 图 8 是Exhaustive 算法和 Idid 有关 TP 值对比情 
况. 实验时采取每 100 个数据包中夹杂 80 个非自体, 其中非自体中有 40 个是刚刚确定的, 即以前这种类 型的 IP 包被认为是自体, 现在被认为是非法的网络 行为, 例如: 紧急关闭其中 40 个端口以停止提供相 关服务.

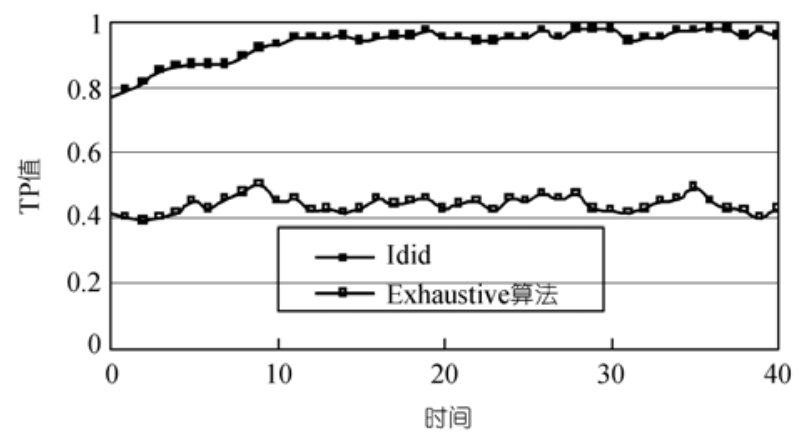

图 8 Exhaustive 和 Idid 的 TP 值比较实验

图 9 显示了 Exhaustive 算法和 Idid 有关 FP 值的 对比情况. 实验时采取每 100 个数据包中夹杂 40 个 自体，其中 20 个自体为新近定义，例如：另外的 20 个网络端口刚被打开以提供新的服务.

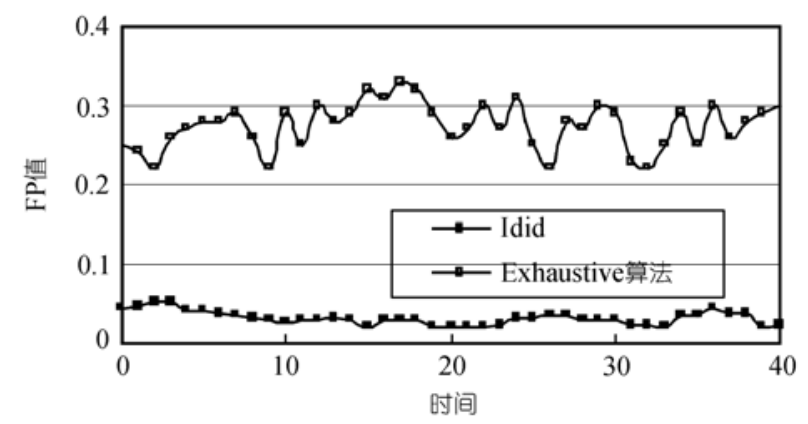

图 9 Exhaustive 和 Idid 的 FP 值比较实验

实验结果表明, Exhaustive 与 Idid 相比有较低的 TP 值和较高的 FP 值, 究其原因为其自体的定义缺乏 灵活性, 不能有效地识别新近加入的自体抗原和非 自体抗原. 与之相反, Idid 通过自体演化和动态耐受 等机制, 避免了免疫细胞对发生变异的自体的耐受, 降低了漏报率, 从而提高了模型的检测率 TP; 与此 同时, 模型通过自体演化和记忆细胞的淘汰机制等, 避免了模型对新加入自体的识别, 从而降低了模型 的误报率 FP.
图 10 显示在大小不同的自体集合情况下, Exhaustive 和 Idid 在成熟细胞生成效率(时间代价)上的 差异, 其中, 需要生成的成熟细胞的数量固定 (20个). 在 Idid中, 由于用于耐受的自体元素数量相对稳定且 数量较少, 因此用于产生固定数量的成熟细胞的时 间代价也相对恒定，而 Exhaustive 算法的时间花费则 随自体元素数目的增加而呈指数增长. 实验结果显 示, Idid 较 Exhaustive 在成熟细胞生成效率方面有较 大的优势.

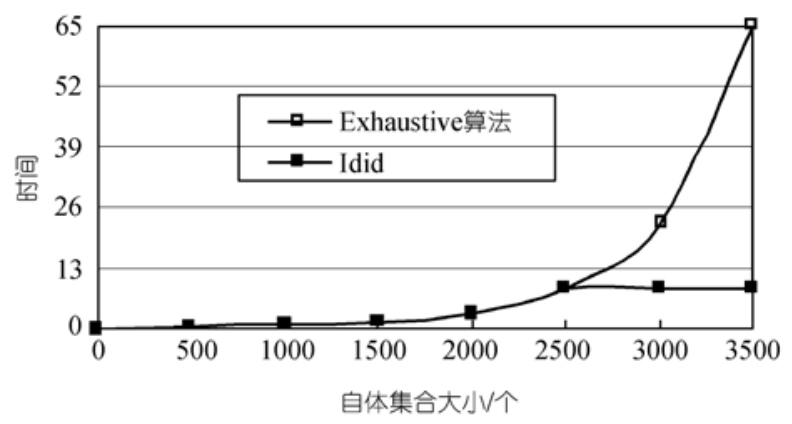

图 10 Exhaustive 算法与 Idid 在成熟免疫细胞生成效率上 的比较实验

\section{3 结论}

入侵检测中, 关于自体的描述是一件非常困难 的工作, 目前已报道的基于免疫的入侵检测系统中 的自体描述，几乎都是静态的，不能适应日益复杂的 网络情况, 缺乏自适应性. 同时, 静态的自体描述经 常导致真实网络环境中一个无法回避的问题：成熟 细胞的训练代价随自体的数目呈指数增长.

本文提出了一种新的自体自然动态定义方法, 通过设置自体的演化周期, 从网上自然收集新的自 体元素, 同时淘汰那些已无太多用处的自体元素(这 些元素长时间不出现), 并引入未成熟细胞的动态耐 受这一新概念, 巧妙地将变与不变有机地统一起来, 从而使自体的定义达到了与真实网络世界随时间同 步动态演化的目的, 同时又从微观上确保了 CIS 的稳 定性, 避免了成熟细胞训练的时空代价呈指数增长 的难题.

另外, Idid采用集合代数定量描述, 其间没有任 何变量以及变量赋值等概念, 只有表达式, 因此, 模 型中所有集合的演化均可并发进行 ${ }^{[20]}$.

1 Idid 模型具有店泛的用途, 例如将自体视为操作 
系统或文件的正常状态，或某种既定模式等随时间 动态变化的外部环境, 合理调整抗原集合, 则该模型 还可以应用于病毒检测及模式识别等领域.

致谢 本工作为国家自然科学基金(批准号: 60373110)、教 育部新世纪优秀人才计划(批准号: NCET-04-0870)、教育部 博士点基金(批准号：20030610003)、四川省科技厅应用基 础基金(批准号：05JY029-021-1)和四川大学创新基金(批准 号: 2004CF10)资助项目.

\section{参考文献}

1 李涛. 网络安全概论. 北京: 电子工业出版社, 2004

2 李涛. 计算机免疫学. 北京: 电子工业出版社, 2004

3 de Castro L N, Timmis J I. Artificial immune systems as a novel soft computing paradigm. Soft Computing Journal, 2003, 7(8): 526 544

4 Spears W M, De Jong K A. An overview of evolutionary computation. Lecture Notes in Computer Science, 1993, 667: 442 459

5 de Castro L N, Timmis J I. Artificial Immune Systems: A New Computational Intelligence Approach. London: Springer-Verlag, 2002

6 李涛. 基于免疫的网络安全风险检测. 中国科学, $\mathrm{E}$ 辑：信息科 学, 2005, 35(8): 798 816

7 焦李成, 杜海峰. 人工免疫系统进展和展望. 电子学报, 2003, 31(10): 1540 1548

8 肖人彬, 王否. 人工免疫系统: 原理、模型、分析及展望. 计算 机学报, 2002, 25(12): 1281 1293

9 Forrest S, Perelson A S. Self-nonself discrimination in a computer. In: IEEE Symposium on Security and Privacy. Oakland, 1994. CA: IEEE Computer Society Press, 1994. 202 213

10 Forrest S, Hofmeyr S, Somayaji A. Computer immunology. CACM, 1997, 40(10): 88 96
11 Hofmeyr S, Forrest S. Immunity by design: an artificial immune system. In: Genetic Evolutionary Computation Conf, San Francisco, 1999. CA: IEEE Press, 1999. 1289 1296

12 Hofmeyr S, Forrest S. Architecture for an artificial immune system. Evolutionary Computation, 2000, 8(4): 443 473[DOI]

13 Dasgupta D. Immunity-based intrusion detection system: A general framework. In: the 22nd National Information Systems Security Conf, Crystal City, 1999. VA: CISSE, 1999. 147 160

14 Harmer P K, Williams P D, Gunsch G H, et al. An artificial immune system architecture for computer security applications. IEEE Transaction on Evolutionary Computation, 2002, 6(3): 252 280[DOI]

15 D'haeseleer P, Forrest S. An immunological approach to change detection: algorithm, analysis and implication. In: IEEE Symposium on Research in Security and Privacy. Oakland, 1996. CA: IEEE Computer Society Press, 1996. 110 119

16 Kim J, Bentley P J. Towards an artificial immune system for network intrusion detection: an investigation of dynamic clonal selection. In: the Congress on Evolutionary Computation (CEC2002), Honolulu, 2002. Piscataway: IEEE Press, 2002. 1015 1020

17 梁可心, 李涛, 刘勇, 等. 一种基于人工免疫理论的新型入侵检 测模型. 计算机工程与应用, 2005, 41(2): 129 132

18 Perelson A S, Weisbuch G. Immunology for physicists. Review of Modern Physics, 1997, 69(4): 1219 1263[DOI]

19 Kim J, Bentley P J. Negative selection: how to generate detectors. In: Timmis J, Bentley P J, eds. The First International Conference on Artificial Immune Systems (ICARIS), Canterbury UK, 2002. Kent: Canterbury Printing Unit, 2002. 89 98

20 Backus J. Can programming be liberated from the Von Neumann Style? A functional style and its algebra of programs. CACM, 1978, 21(8): 613 641

(2005-01-11 收稿, 2005-08-10 收修改稿) 